\title{
Bearing Fault Detection of IPMSMs using Zoom FFT
}

\author{
June-Seok Lee*, Tae-Min Yoon** and Kyo-Beum Lee ${ }^{\dagger}$
}

\begin{abstract}
This paper presents a bearing fault detection method for permanent magnet synchronous motors (PMSMs). Owing to its good characteristics, PMSM usage has increased; however, a substantial number of motor failures are also reported. Many studies have focused on bearing fault detection using vibration sensors. However, current sensors are already installed in many industries, and therefore, if bearing faults can be detected using these sensors, there would be no need to install additional sensors. A frequency analysis is performed to detect bearing faults and fast Fourier transform (FFT)-based methods can be used for the same. FFT needs to have a high resolution to be able to differentiate between the frequencies of bearing faults from those of the stator current. However, FFT requires extensive data and high computational cost to achieve this high resolution. Therefore, the zoom FFT (ZFFT) algorithm is implemented to minimize the computational cost and to increase the resolution. The experimental results verify the effectiveness of the proposed method by comparing FFT and ZFFT waveforms.
\end{abstract}

Keywords: Bearing fault detection, Permanent magnet synchronous motor, Frequency analysis, Zoom FFT

\section{Introduction}

Permanent magnet synchronous motors (PMSMs) are used in a variety of applications. Fig. 1 shows a generalized structure of an IPMSM. Because the rotor of the PMSM is a permanent magnet, it does not require an external power source to generate the flux. This helps minimize power consumption and improve system efficiency $[1,2]$. Thus, PMSMs compare well to other motors. As PMSM usage increases, motor failures are also expected. Accordingly, several researchers have conducted motor reliability studies. The results of several studies show that bearing faults cause more than $40 \%$ of all machine failures [3]. Thus, it is critical to monitor the condition of bearings in electrical machines.

In many studies, a popular approach has been to measure bearing condition using a vibration sensor. This is because bearing faults cause radial movement between the stator and rotor, and bearing fault characteristics are reflected in the machine vibration [4]. However, an accelerometer or vibration velocity transducer has to be attached to the bearing or bearing support housing directly. Further, additional system cost is incurred because of the additional vibration sensor.

As a result, monitoring bearing condition through the stator current has been studied for many decades. Many researchers have determined that motor vibration frequencies

$\dagger$ Corresponding Author: Dept. of Electrical and Computer Engineering, Ajou University, Suwon, Korea. (kyl@ajou.ac.kr).

* Railroad Safety Research Division, Korea Railroad Research Institute, Uiwang, Korea. (ljs@krri.re.kr).

** LG electronics, Gasan, Korea. (taemin.yoon@lge.com)

Received: March 1, 2015; Accepted: April 14, 2016 are reflected in the stator current [5-7]. Therefore, monitoring the stator current provides information about bearing faults. In general, current sensors are already installed in many industries and therefore, there is no need to install additional sensors to monitor the stator current. Thus, monitoring the stator current to detect bearing faults provides economic and implementation benefits.

Frequency analysis of the stator current has to be performed to detect frequencies through bearing faults. The traditional frequency analysis method is the fast Fourier transform(FFT). The FFT can provide frequency information via a simplified Fourier transform algorithm. However, there are several frequencies in the stator current and it is difficult to differentiate between these frequencies and those of the bearing faults [8]. Thus, it is necessary to increase the resolution of the FFT to detect the bearing fault frequencies. Because the resolution of the FFT depends on the sampling frequency, when the number of

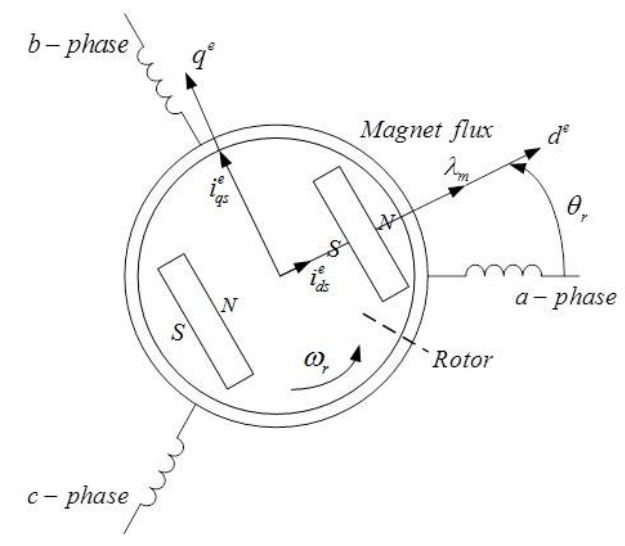

Fig. 1. Equivalent model of IPMSMs 
samples is the same, FFT-based methods require a large amount of data and high computational costs to increase the resolution. However, it is not always possible to satisfy the requirement of extensive data because of hardware limitations.

The short time Fourier transform (STFT) can be used to achieve robustness and simplicity to overcome the limitations of the FFT [9]. However, the STFT is dependent on the type and the length of the window, and therefore, a tradeoff between time and frequency resolution is needed.

In fact, because the frequency of the bearing fault is present in specific frequency bins, and the high frequency resolution and narrow frequency band are needed, it is more efficient to expand the frequency region of interest. Therefore, the zoom FFT (ZFFT) algorithm has been studied to decrease the amount of data and computational cost $[10,11]$. The ZFFT is the preferred method to magnify the frequency band centered on an arbitrary frequency. Therefore, the ZFFT can increase frequency resolution while keeping the same sampling frequency and number of samples.

This paper presents an efficient method to detect bearing fault by monitoring the stator current using ZFFT analysis. To generate bearing faults and scrutinize the validity of the ZFFT, equipment for generating oscillation is used. Because the ZFFT is efficient regarding the computational cost, the proposed method can give effective way to find out the bearing fault.

\section{Stator Current Spectral Analysis}

There are several reasons that can cause bearing faults. For example, an installation problem can cause the bearing to be unbalanced on the shaft or in the housing. Fig. 2 shows four types of bearing misalignments. Further, a prolonged period of motor drive leads to abrasion in bearings that produce motor vibration. Since ball bearings support the rotor, any bearing fault or unbalance causes a radial movement to the rotor and the stator.

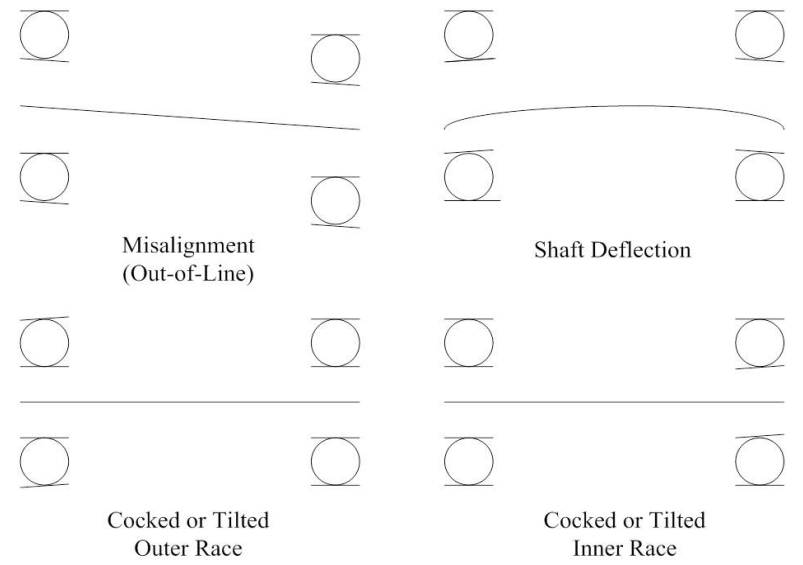

Fig. 2. Four types of rolling-element bearing misalignment
The relationship between the bearing vibration and the stator current can be described by the air gap eccentricity. Because of the air gap eccentricity, anomalies are produced in the air gap flux density and the predictable frequencies are reflected in the stator current. These predictable frequencies, $f_{b n g}$, are given by [3]

$$
f_{\text {bng }}=\left|f_{f} \pm m f_{c f}\right| \text {, }
$$

where $m=1,2,3, \cdots, f_{f}$ is the fundamental frequency and $f_{c f}$ is one of the characteristic bearing fault frequencies.

The characteristic frequencies for a bearing fault depend on bearing dimensions as shown in Fig. 3. These frequencies can be described by three practicable forms [3]. First, the ball spin frequency is produced by the rotation of each ball. Since the ball makes contact with the inner and outer raceway upon each rotation, the vibration frequency in the stator current is twice the spin frequency and is given by

$$
f_{b s}=\frac{P D}{B D} f_{m r}\left[1-\left(\frac{B D}{P D} \cos \beta\right)^{2}\right] \text {, }
$$

where PD is the bearing pitch diameter, $\mathrm{BD}$ is the ball diameter, $\beta$ is the contact angle of the balls on the races and $f_{m r}$ is the mechanical rotor speed in Hertz.

Next, the outer and inner raceway frequencies need to be identified. These frequencies are produced when each ball touches a defect in the outer or inner raceway. If the number of balls is $n$, the vibration occurs n-times in the total cycle. Therefore, the outer and inner raceway frequencies are described as

$$
f_{o}=\frac{n}{2} f_{m r}\left[1-\frac{B D}{P D} \cos \beta\right]
$$

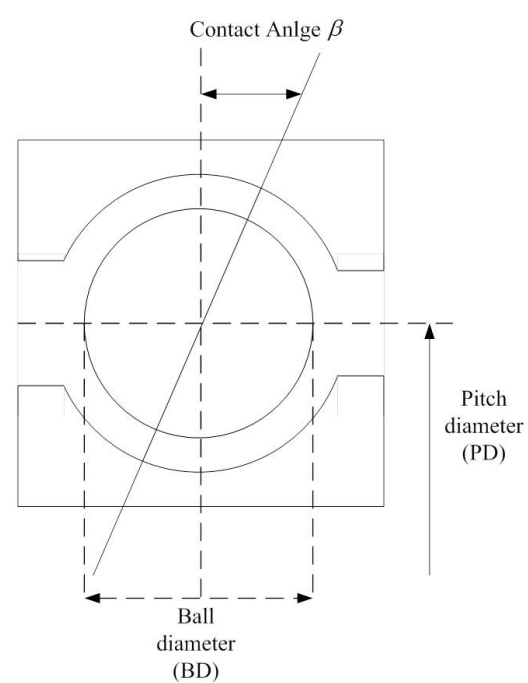

Fig. 3. Ball bearing dimensions 


$$
f_{i}=\frac{n}{2} f_{m r}\left[1+\frac{B D}{P D} \cos \beta\right] .
$$

The three practicable forms of bearing fault frequency - (2), (3), and (4) — require bearing dimensions to calculate the exact result. However, the characteristic frequencies can be approximated with forms where bearing dimensions are unnecessary [3].

$$
\begin{aligned}
& f_{o}=0.4 \times n \times f_{m r} \\
& f_{i}=0.6 \times n \times f_{m r} .
\end{aligned}
$$

These simplified equations can be used for bearings between six and twelve balls and allow users to detect bearing faults without accurate understanding about bearing dimensions. Therefore, bearing fault analysis can be performed easily by monitoring the stator current.

\section{Basic Theory of ZFFT}

\subsection{Theory of ZFFT}

As stated in section II, the characteristic bearing fault frequency is predictable. For frequency analysis of the stator current, FFT-based methods can be implemented. However, the magnitude of these frequencies is much less compared to that of other frequencies, such as a cyclical load torque variation and the fundamental frequency [8]. In addition, because the bearing fault frequencies are already known, a specific frequency band is only necessary to detect the bearing fault. Accordingly, a high-resolution frequency analysis technique is required to distinguish these frequencies.

To increase resolution in the FFT, the sampling time and the number of samples need to be increased. However, these methods can lead to an increase in the computational cost. Further, if we only need the particular frequency region and high resolution, FFT-based methods require unnecessarily excessive calculations. The ZFFT algorithm has been studied taking this into consideration.

The ZFFT is a useful algorithm to expand the narrow frequency band centered on any frequency. This is closely related to multi-rate filtering and is the same compared to the single sideband modulation [12]. In real applications on DSP-based systems, memory limitations exist and the size of matrices in the FFT prevents augmenting the number of samples. Compared with FFT, the ZFFT algorithm can provide a direct reduction of sampling frequency while maintaining the same sampling time and number of samples, thus increasing the frequency resolution.

A process of the ZFFT algorithm is shown in Fig. 4. Let us consider the system of $\mathrm{N}$ samples and the sampling frequency, $\mathrm{f}_{\text {samp }}$, to compare the resolution between the ZFFT and the FFT.

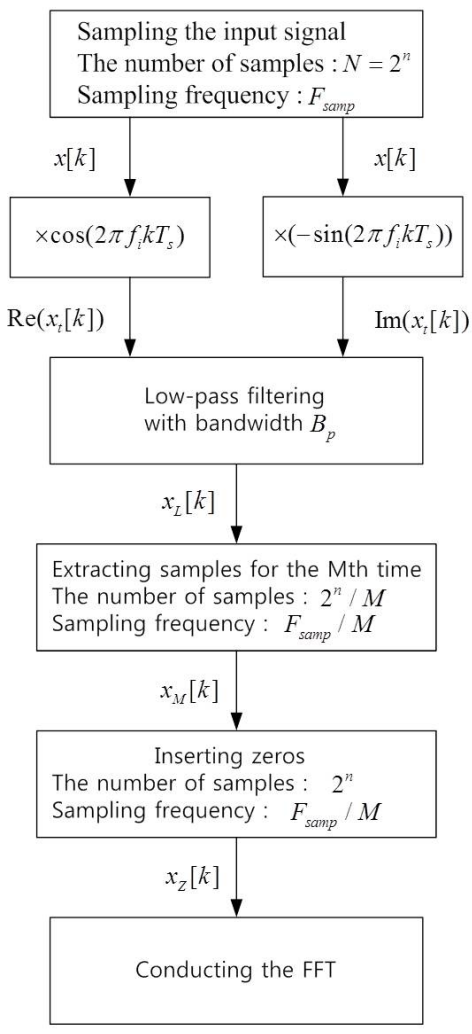

Fig. 4. Block diagram of the ZFFT process

First, because the FFT is calculated on the condition of the power of two, the input signal is sampled at $N=2^{n}$. Then, the frequency resolution is $\Delta f=\mathrm{f}_{\text {samp }} / N$. The frequency transition is performed to expand a narrow span centered on interested frequency $f_{c}$. This process can be conducted by multiplying the real number by $\cos \left(2 \pi f_{c} t\right)$ and the complex number by $\sin \left(2 \pi f_{c} t\right)$. This is expressed as

$$
\begin{aligned}
& x[k] \exp \left(j 2 \pi f_{c} t\right) \leftrightarrow X\left(f-f_{c}\right) \\
& x[k] \exp \left(j 2 \pi f_{c} t\right)=x[k]\left[\cos \left(2 \pi f_{c} t\right)+j \sin \left(2 \pi f_{c} t\right)\right]
\end{aligned}
$$

The signal $x[k]$ is obtained after the process of sampling the signal. The complex signal after the frequency transition is filtered by a low-pass filter with a bandwidth $B_{p}$ and $\left[f_{c}, f_{c}+B_{p}\right]$ is the frequency band of interest.

Second, the filter output signal $x_{L}[k]$ is undersampled. This process is called decimation. In this process, the signal $x_{L}[k]$ is extracted for the Mth time where $\mathrm{M}$ is a decimation factor. Owing to the Mth time sampling, the number of samples reduces to $N / M$ and the sampling frequency also reduces to $\mathrm{f}_{\text {samp }} / M$. Therefore, the frequency resolution is $\Delta f=\left(\mathrm{f}_{\text {samp }} / M\right) /(N / M)$ and this is the same frequency resolution of that of the first process.

Finally, the decimation signal $x_{M}[k]$ is filled with zeros at the end of this signal in the time domain. This process is called zero padding. The number of zeros that fills the 
matrix of $x_{M}[k]$ is the number of signals reduced in the decimation process, that is $N-(N / M)$ and can be described as

$$
\begin{aligned}
K & =\frac{[\text { The number of zeros }]}{[\text { The number of samples in the decimation }]}, \\
& =\frac{[N-(N / M)]}{N / M}
\end{aligned}
$$

where $\mathrm{K}$ is the zero padding factor.

If zeros of the zero padding process are filled with the end of a matrix $x_{M}[k]$ in the time domain, zeros of $\mathrm{K}$ are inserted in the intervals of each sample in the frequency domain. As a result, the number of inserted zeros does not affect the sampling, the sampling frequency is $\mathrm{f}_{\text {samp }} / M$ identically and the frequency resolution is $\Delta f=$ $\left(\mathrm{f}_{\text {samp }} / M\right) / N$. Thus, it is evident that the ZFFT increases the resolution $\mathrm{M}$ times more than the FFT.

When the ZFFT and FFT have the same frequency resolution, $\mathrm{N}_{\text {ZFFT }}$ and $\mathrm{N}_{\mathrm{FFT}}$ required to the ZFFT and FFT are calculated as

$$
\begin{aligned}
& N_{Z F F T}=\frac{\mathrm{f}_{\text {samp }} / M}{\Delta f} \\
& N_{F F T}=\frac{\mathrm{f}_{\text {samp }}}{\Delta f}
\end{aligned} .
$$

The computation (C) comparison between the ZFFT and FFT is represented as

$$
\begin{aligned}
& C_{Z F F T}=N_{Z F F T} \log _{2} N_{Z F F T}=\frac{\mathrm{f}_{\text {samp }} / M}{\Delta f} \log _{2} \frac{\mathrm{f}_{\text {samp }} / M}{\Delta f} \\
& C_{F F T}=N_{F F T} \log _{2} N_{F F T}=\frac{\mathrm{f}_{\text {samp }}}{\Delta f} \log _{2} \frac{\mathrm{f}_{\text {samp }}}{\Delta f}
\end{aligned}
$$

Depending on the value of $\mathrm{M}, \mathrm{C}_{\mathrm{ZFFT}}$ can become smaller than $\mathrm{C}_{\mathrm{FFT}}$.

\subsection{Application of Equipment for Bearing Fault}

Bearing faults are classified into single-point defects and generalized roughness faults. Single-point defects are defined as a single, localized defect on an otherwise relatively undamaged bearing surface, for example, a pit or spall [7]. Under single-point defects, the characteristic bearing fault frequencies are produced depending on which surface of the bearing contains the faults as described in section II.

Generalized roughness faults are a type of fault where the condition of a bearing surface has degraded considerably over a large area and has become rough, irregular, or deformed, for example the overall surface roughness produced by a contamination or loss of lubricant [7]. These

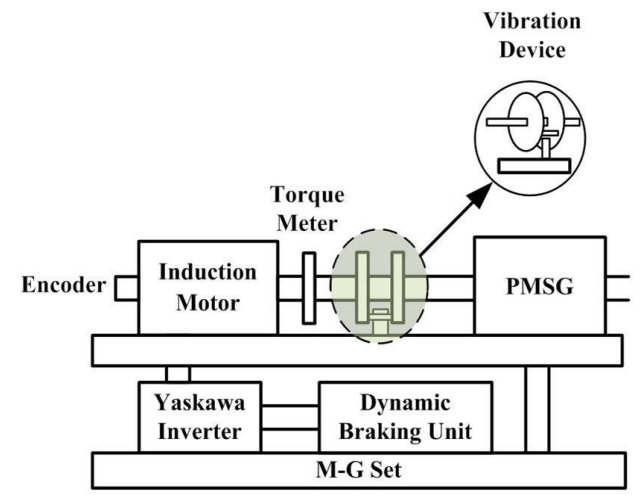

Fig. 5. Model of the bearing fault equipment

generalized roughness faults do not exhibit the normal frequency characteristic as that of the characteristic bearing fault frequencies. Since they are difficult to identify, many studies are being conducted to identify these faults.

Therefore, in this study, the equipment for producing bearing faults are installed to prove the validity of the ZFFT algorithm for detecting bearing faults under singlepoint defects. Fig. 5 shows a model of the equipment for producing bearing faults. As shown in Fig. 5, four bars are installed at the axis of rotation of a motor and an obstacle is raised under the bars so that harmonic frequencies are produced when the motor rotates. The mechanical rotation frequency is based on the motor speed in rpm as

$$
f_{m}=\omega_{r p m} \times \frac{P}{120},
$$

where $\mathrm{P}$ is the number of poles.

The harmonic frequencies of the line current produced by an obstacle are the quadruple frequency of the mechanical rotation frequency calculated in (11) and the multiple harmonics of the quadruple frequency as described in (12) and (13).

$$
\begin{gathered}
f_{h}=f_{m}+m \times f_{o s c} \\
f_{o s c}=4 \times f_{m},
\end{gathered}
$$

where $f_{\text {osc }}$ is the quadruple frequency of the mechanical rotation frequency.

The harmonic frequencies of the line current produced by an obstacle in (12) have the same form as the characteristic bearing fault frequencies generated by a single-point defect described from (1) to (4). Thus, the validity of the ZFFT algorithm for detecting bearing faults under single-point defects can be proved through the equipment.

In this study, the ZFFT algorithm is conducted via the qaxis current in a synchronous reference frame. Because oscillation is produced by the axis of rotation and the torque is related to the q-axis current, the quadruple frequency of the mechanical rotation frequency can be detected by the q-axis current without considering the 
mechanical rotation frequency.

\section{Experimental Results}

The experiment is implemented using a digital signal processor (DSP) TMS320F28335. The model of the equipment for producing bearing faults is realized as Fig. 6 . To test the proposed method for bearing fault detection, bearing fault signals are generated from the equipment as the motor rotates at $50 \mathrm{rpm}$. Line currents of the motor are measured by current sensors to monitor the harmonic frequencies and the amplitude spectrum is calculated to show the result of the FFT and ZFFT. The exact parameters of the bearing fault signals are shown in Table 1 .

From (12) and (13), the quadruple frequency of the mechanical rotation frequency is $10 / 3 \mathrm{~Hz}$ and its first harmonic frequency is $20 / 3 \mathrm{~Hz}$. Because the frequency resolution is $0.25 \mathrm{~Hz} / \mathrm{sample}$, the quadruple frequency of the mechanical rotation frequency and its first harmonic frequency will appear at the 13th and 26th samples, respectively.

Since ordinal numbers of the bearing fault signal can be calculated, bearing faults can be detected. However, the magnitude of bearing fault frequency is unknown and it is possible that unrelated noises exist at the corresponding bearing fault frequency. Therefore, when an ordinal number of the bearing fault frequency is derived from (12) and (13), the proposed method monitors at a corresponding ordinal number and calculates the average value of its magnitude during a set period.

Fig. 7 shows the waveform of the FFT. Experiment parameters are the same as that in Table 1 to compare the waveform with the ZFFT. The FFT is conducted using an a-phase current and the process of frequency transition is

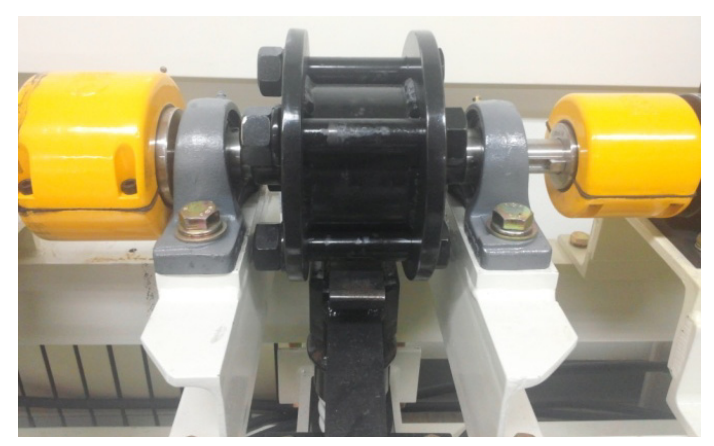

Fig. 6. Experiment setup of the bearing fault equipment

Table 1. Motor specifications

\begin{tabular}{c|c}
\hline Motor speed & $50 \mathrm{rpm}$ \\
\hline Number of poles & 6 \\
\hline Mechanical rotation frequency & $5 / 6 \mathrm{~Hz}$ \\
\hline Number of samples & 512 \\
\hline Sampling frequency & $512 \mathrm{~Hz}$ \\
\hline Decimation factor & 4 \\
\hline
\end{tabular}

applied. The quadruple frequency of the mechanical rotation frequency appears at the 3rd sample and its harmonic frequencies appear at the 7th and 10th samples. Because the frequency resolution of the FFT is $1 \mathrm{~Hz} / \mathrm{sample}$, the quadruple frequency and its harmonic frequencies are calculated at $3 \mathrm{~Hz}, 7 \mathrm{~Hz}$, and $10 \mathrm{~Hz}$. Therefore, the errors of each frequency are approximately $0.33 \mathrm{~Hz}, 0.33 \mathrm{~Hz}$, and $0 \mathrm{~Hz}$. This is because the 10th sample, $30 / 3 \mathrm{~Hz}$ can be divided by the frequency resolution which is $1 \mathrm{~Hz} /$ sample and the other samples, $10 / 3 \mathrm{~Hz}$ and $20 / 3 \mathrm{~Hz}$, cannot be divided.

Fig. 8 shows the waveform of the ZFFT. The ZFFT is also conducted for an a-phase current. The quadruple frequency of the mechanical rotation frequency is shown at the 13th sample and its harmonic frequencies appear at the 26 th and 40th samples. Because the frequency resolution is $4 \mathrm{~Hz} /$ sample, the quadruple frequency and its harmonic frequencies are each calculated at $13 / 4 \mathrm{~Hz}, 26 / 4 \mathrm{~Hz}$, and $40 / 4 \mathrm{~Hz}$. The errors of each frequency are approximately $0.08 \mathrm{~Hz}, 0.16 \mathrm{~Hz}$, and $0 \mathrm{~Hz}$. Therefore, the ZFFT is more accurate than the FFT when the number of samples and the sampling frequency are the same.

Fig. 9 shows waveforms of the q-axis current and its frequency analysis on an oscilloscope. Because of the quadruple frequency of the mechanical rotation frequency

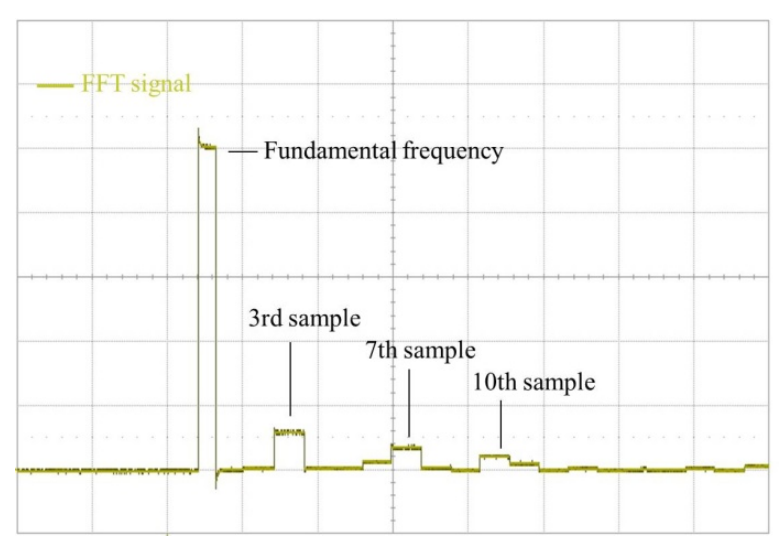

Fig. 7. FFT spectrum of a-phase current

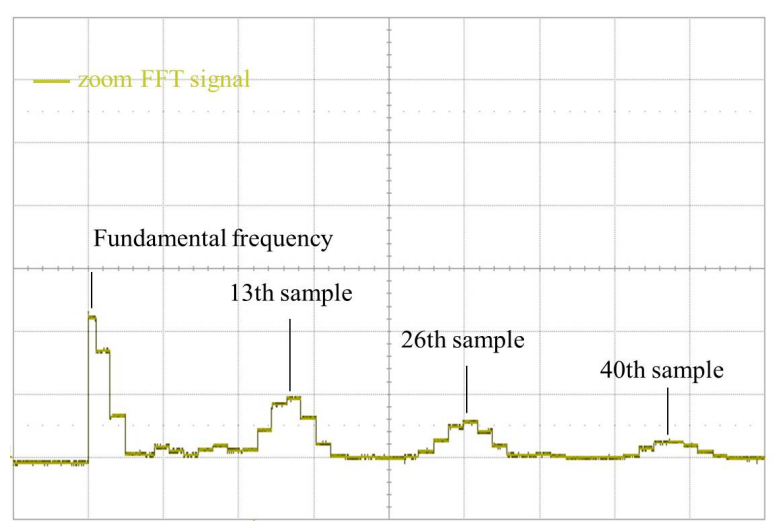

Fig. 8. ZFFT spectrum of a-phase current 
by the equipment for producing bearing faults, oscillation is shown in the q-axis current. The expected harmonic frequencies that are the quadruple frequency and its multiple harmonic frequencies from (12) and (13) can be detected by FFT with an oscilloscope and the magnitude of multiple harmonic frequencies decreases as the frequency of those increases.

Fig. 10 shows waveforms of the a-phase current and ZFFT of the q-axis current. The sampling period shows the number of total samples, which is 512 in this experiment. In the waveform of the ZFFT, multiple harmonic frequencies appear as described above. The first frequency of the ZFFT is the quadruple frequency of the mechanical rotation frequency and its ordinal number is 13 . The other frequencies are the harmonic frequencies of the quadruple frequency. The ordinal numbers of each frequency are 26 , 40 , and 52, and those frequencies calculated by frequency resolution are $20 / 3 \mathrm{~Hz}, 30 / 3 \mathrm{~Hz}$, and $40 / 3 \mathrm{~Hz}$. Therefore, frequency analysis of the ZFFT is accurate.

Fig. 11 shows waveforms of the a-phase current under no-load and load conditions and the ZFFT of the q-axis current for the healthy machine. The a-phase current is constant and there are no frequencies except noise in the ZFFT spectrum.

Fig. 12 shows waveforms of the a-phase current under no-load and load conditions and the ZFFT of the q-axis current for the faulty machine. The a-phase current changes with the motor oscillation and there are harmonic frequencies at $10 / 3 \mathrm{~Hz}, 20 / 3 \mathrm{~Hz}$, and $30 / 3 \mathrm{~Hz}$. The amplitude of the ZFFT under load condition for the faulty machine is smaller than that of the ZFFT under no load condition. This is because the fault signals in the q-axis current are related to torque and the load is applied in the opposite direction.

Table 2 shows the computational time of the ZFFT and FFT measured at the experiment. The ZFFT and FFT are the same frequency resolution. Since M of the ZFFT is 4 , the ZFFT has the short computational time four times longer than the FFT.

Table 2. Computational time of ZFFT and FFT

\begin{tabular}{c|c}
\hline ZFFT & FFT \\
\hline 4 us & 16 us \\
\hline
\end{tabular}

\section{Conclusion}

This paper proposes the bearing faults detection method using ZFFT algorithm. The ZFFT algorithm is very suitable to detect the bearing faults because it analyzes frequencies in narrow frequency band with high frequency resolution which corresponds to requirements for detecting the bearing faults. Additionally, in comparison with the FFT algorithm, the ZFFT algorithm needs smaller data and computational cost which are shown in experimental results. Therefore, the proposed method using ZFFT algorithm can be applied with motor control algorithms at a DSP unit.

In experiment, the equipment for producing bearing faults is designed to prove the validity of the ZFFT algorithm. The harmonic frequencies caused by the equipment have the same form as characteristic bearing fault frequencies. According to the experimental results, we know that the proposed method exactly detects these fault frequencies and those results shows validity of the proposed bearing faults detection.

\section{Acknowledgment}

This work was supported in part by the Basic Science Research Program through the National Research Foundation of Korea (NRF) funded by the Ministry of Education (No. 2013R1A1A2A10006090) and in part by the R\&D Program of the Korea Railroad Research Institute, Republic of Korea

\section{References}

[1] Yi-Kyu Kang, Hae-Gwang Jeong, Kyo-Beum Lee, Dong-Choon Lee, and Jang-Mok Kim, "Simple Estimation Scheme for Initial Rotor Position and Inductances for Effective MTPA-Operation in WindPower Systems using an IPMSM," Journal of Power Electronics, vol. 10, no. 4, pp. 396-404, July 2010.

[2] Hyun-Woo Sim, June-Seok Lee, and Kyo-Beum Lee, "On-line Parameter Estimation of Interior Permanent Magnet Synchronous Motor Using an Extended Kalman Filter," Journal of Electrical Engineering \& Technology, vol. 9, no. 2, pp. 600-608, March 2014.

[3] Randy R. Schoen, Thomas G. Habetler, Farrukh Kamran, and Robert G. Bartheld, "Motor Bearing Damage Detection Using Stator Current Monitoring," IEEE Transactions on Industry Applications, vol. 31, no. 6, November/December 1995.

[4] Bo Li, Mo-Yuen Chow, Yodyium Tipsuwan, and James C. Hung, "Neural-network based motor rolling bearing fault diagnosis," IEEE Transactions on Industrial Electronics, vol. 47, no. 5, pp. 1060-1068, October 2000.

[5] Subhasis Nandi, Hamid A. Toliyat, and Xiaodong Li, "Condition Monitoring and Fault Diagnosis of Electrical Motors-A Review," IEEE Transactions on Energy Conversion, vol. 20, no. 4, pp. 719-729, December 2005.

[6] Fabio Immovilli, Alberto Bellini, Riccardo Rubini, and Carla Tassoni, "Diagnosis of Bearing Faults in Induction Machines by Vibration or Current Signals : A Critical Comparison," IEEE Transactions on 
Industry Application, vol. 46, no. 4, pp. 1350-1359, July/August 2010.

[7] Jason R. Stack, Thomas G. Habetler, and Ronald G. Harley, "Fault Classification and Fault Signature Production for Rolling Element Bearing in Electric Machines," IEEE Transactions on Industry Application, vol. 40, no. 3, pp. 735-739, May/June 2004.

[8] Jason R. Stack, Thomas G. Habetler, and Ronald G. Harley, "Bearing Fault Detection via Autoregressive Stator Current Modeling," IEEE Transactions on Industry Application, vol. 40, no. 3, pp. 740-747, May/June 2004.

[9] Birsen Yazici, and Gerald B. Kliman, "An Adaptive Statistical Time-Frequency Method for Detection of Broken Bars and Bearing Faults in Motors Using Stator Current," IEEE Transactions on Industry Application, vol. 35, no. 2, pp. 442-452, March/April 1999.

[10] P. C. Y. YIP, "Some Aspects of the Zoom Transform," IEEE Transactions on Computers, vol. C-25, no. 3, pp. 287-296, March 1976.

[11] Elmer A. Hoyer, and Ronald F. Stork, "The zoom FFT using complex modulation," IEEE International Conference on Acoustics, Speech, and Signal Processing, vol. 2, pp. 78-81, 1977.

[12] Alberto Bellini, Amine Yazidi, Fiorenzo Filippetti, Claudio Rossi, Gerard-Andre Capolino, "High Frequency Resolution Techniques for Rotor Fault Detection of Induction Machines," IEEE Transactions on Industrial Electronics, vol. 55, no. 12, pp. 4200-4209, December 2008.

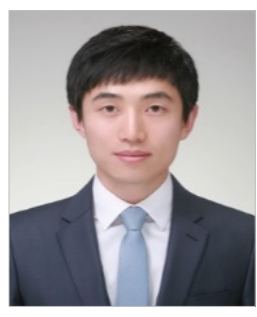

June-Seok Lee received the B.S., M.S., and $\mathrm{Ph} . \mathrm{D}$. degrees in electrical and computer engineering from Ajou University, Suwon, Korea, in 2011, 2013, and 2015, respectively. Since 2015, he has been with the Korea Railroad Research Institute, Uiwang, Korea. His research interests include grid-connected systems, multilevel inverter and reliability.

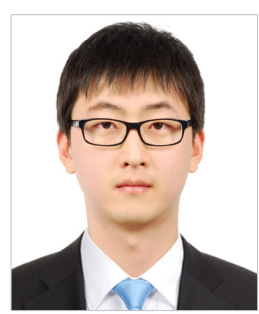

Tea-Min Yoon received the B.S and M.S degree in electrical and computer engineering from Ajou University, Korea, in 2013 and 2015. He is currently working for LG electronics. His research interests include electric machine drives.

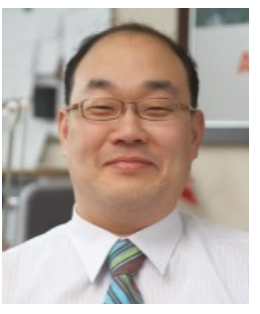

Kyo-Beum Lee received the B.S. and M.S. degrees in electrical and electronic engineering from the Ajou University, Korea, in 1997 and 1999, respectively. $\mathrm{He}$ received the $\mathrm{Ph} . \mathrm{D}$. degree in electrical engineering from the Korea University, Korea in 2003. From 2003 to 2006, he was with the Institute of Energy Technology, Aalborg University, Aalborg, Denmark. From 2006 to 2007, he was with the Division of Electronics and Information Engineering, Chonbuk National University, Jeonju, Korea. In 2007 he joined the School of Electrical and Computer Engineering, Ajou University, Suwon, Korea. He is an associated editor of the IEEE Transactions on Power Electronics, the Journal of Power Electronics, and the Journal of Electrical Engineering \& Technology. His research interests include electric machine drives, renewable power generations, and electric vehicle applications. 